\title{
Efficacy of Tofacitinib for the Treatment of Psoriatic Arthritis: Pooled Analysis of Two Phase 3 Studies
}

\author{
Peter Nash • Laura C. Coates - Roy Fleischmann - Kim A. Papp • \\ Juan Jesus Gomez-Reino · Keith S. Kanik • Cunshan Wang • \\ Joseph Wu · Sujatha Menon · Thijs Hendrikx • William C. Ports
}

Received: August 9, 2018 / Published online: November 9, 2018

(C) The Author(s) 2018

\section{ABSTRACT}

Introduction: Tofacitinib is an oral Janus kinase inhibitor for the treatment of psoriatic arthritis (PsA). This post hoc analysis assessed the efficacy of tofacitinib using pooled data from two phase 3 studies of patients with active PsA.

Methods: Data were pooled from OPAL Broaden (NCT01877668) and OPAL Beyond (NCT01882439). Patients had active PsA and either an inadequate response (IR) to

Enhanced Digital Features To view enhanced digital features for this article go to https://doi.org/10.6084/ m9.figshare.7207310.

Electronic supplementary material The online version of this article (https://doi.org/10.1007/s40744018-0131-5) contains supplementary material, which is available to authorized users.

P. Nash $(\bowtie)$

Department of Medicine, University of Queensland, St Lucia, Brisbane, Australia

e-mail: drpnash@tpg.com.au

L. C. Coates

Nuffield Department of Orthopaedics,

Rheumatology and Musculoskeletal Sciences,

University of Oxford, Oxford, UK

R. Fleischmann

Metroplex Clinical Research Center and University of Texas Southwestern Medical Center, Dallas, TX, USA $\geq 1$ conventional synthetic disease-modifying antirheumatic drug (csDMARD) and were tumor necrosis factor inhibitor (TNFi)-naïve (OPAL Broaden), or had IR to $\geq 1$ TNFi (OPAL Beyond). Pooled data included tofacitinib 5 or $10 \mathrm{mg}$ twice daily (BID; to month 6) and placebo (to month 3; patients then switched to tofacitinib 5 or $10 \mathrm{mg}$ BID). Patients also received one background csDMARD. Endpoints included American College of Rheumatology (ACR) 20 response and change from baseline in Health Assessment Questionnaire-Disability Index (HAQ-DI) at month 3 (primary endpoints), ACR50/70 response, HAQ-DI response (decrease from baseline $\geq 0.35$ ) and improvements in painful and swollen joint counts, psoriasis, enthesitis and dactylitis to month 6 .

Results: A total of 710 patients were included (tofacitinib $5 \mathrm{mg}$ BID: 238; tofacitinib $10 \mathrm{mg}$ BID: 236; placebo: 236). Primary endpoints

\author{
K. A. Papp \\ Probity Medical Research and K Papp Clinical \\ Research Inc, Waterloo, ON, Canada

\section{J. J. Gomez-Reino} \\ Fundación Ramón Domínguez, Hospital Clinico \\ Universitario, Santiago de Compostela, Spain \\ K. S. Kanik · C. Wang · J. Wu · S. Menon · \\ W. C. Ports \\ Pfizer Inc, Groton, CT, USA \\ T. Hendrikx \\ Pfizer Inc, Collegeville, PA, USA
}


showed significant improvements at month 3 in patients receiving tofacitinib 5 or $10 \mathrm{mg}$ BID vs. placebo. Significant improvements in HAQ-DI response, painful and swollen joints, psoriasis, enthesitis and dactylitis vs. placebo were observed for both tofacitinib doses at month 3 . Efficacy was maintained to month 6 (final pooled time point).

Conclusions: In a pooled analysis of csDMARDIR/TNFi-naïve and TNFi-IR patients, tofacitinib was superior to placebo at month 3 across four PsA domains: peripheral arthritis, psoriasis, enthesitis and dactylitis.

Trial Registration: OPAL Broaden (NCT01877668); OPAL Beyond (NCT01882439).

Funding: Pfizer Inc.

Keywords: Janus kinase inhibitor; Psoriatic arthritis; Spondyloarthritis; Tofacitinib; Treatment

\section{INTRODUCTION}

Psoriatic arthritis (PsA) is a chronic inflammatory disease that can impact multiple domains, including peripheral arthritis, skin and nail psoriasis, enthesitis, dactylitis, and spondylitis [1]. PsA occurs in approximately $20-30 \%$ of patients with psoriasis [2-4], and can be associated with substantial healthcare costs, impairments in health-related quality of life, and work productivity [5-7].

Although there are efficacious treatments for PsA currently approved, not all patients achieve satisfactory disease control as evidenced by their failure to attain an American College of Rheumatology (ACR) 20 response after 24 weeks in randomized clinical trials [8-13]. A number of studies report that over $50 \%$ of patients treated with tumor necrosis factor inhibitor (TNFi) therapy for up to 12 months fail to reach minimal disease activity [14-17]. Due to the inability of any approved medication to treat all patients effectively, approximately $50 \%$ of patients have been reported to switch, restart after a treatment gap, or discontinue therapy within the first year of treatment in the United States [18], strongly suggesting that there is a significant unmet need for new therapies with novel mechanisms of action for patients with PsA. Research into the proinflammatory mechanisms of the pathogenesis of PsA has resulted in the development of small molecule therapies for the treatment of PsA, including apremilast and tofacitinib [19].

Tofacitinib is an oral Janus kinase inhibitor for the treatment of PsA. The safety and efficacy of tofacitinib 5 and $10 \mathrm{mg}$ twice daily (BID) have been demonstrated in phase 3 trials of 6 and 12 months' duration in patients with active PsA and an inadequate response (IR) to conventional synthetic disease-modifying antirheumatic drugs (csDMARDs) or TNFi therapy [20-22]. Tofacitinib is also being investigated in an ongoing long-term extension (LTE) study in patients with PsA (NCT01976364).

Pooling data from clinical studies of patients with PsA offers a larger patient sample size for the analysis of disease manifestations that do not affect all patients with PsA, such as enthesitis, dactylitis, axial involvement, and current psoriasis, and yields more precise estimates for endpoints that assess these manifestations compared with the individual studies. This post hoc analysis reports the efficacy of tofacitinib using pooled data from the two pivotal phase 3 studies of patients with PsA.

\section{METHODS}

\section{Study Design}

Data from baseline to month 6 were pooled from patients participating in the two phase 3 studies who had been randomized to tofacitinib 5 or $10 \mathrm{mg}$ BID (for the duration of the study) or placebo.

The Oral Psoriatic Arthritis trial (OPAL) Broaden (A3921091; NCT01877668) was a 12-month, global, double-blind, doubledummy, placebo- and active-controlled parallelgroup phase 3 study in TNFi-naïve adults with active PsA receiving one background csDMARD and with an IR to $\geq 1$ csDMARD. Patients were randomized 2:2:2:1:1 to receive tofacitinib $5 \mathrm{mg}$ BID, tofacitinib $10 \mathrm{mg}$ BID, an active comparator (adalimumab $40 \mathrm{mg}$ subcutaneously once every other week), placebo $\rightarrow$ tofacitinib $5 \mathrm{mg}$ 
BID or placebo $\rightarrow$ tofacitinib $10 \mathrm{mg}$ BID. Patients on placebo switched to tofacitinib at month 3 [20].

OPAL Beyond (A3921125; NCT01882439) was a 6-month, global, double-blind, placebocontrolled, parallel-group phase 3 study in adults with active PsA receiving one background csDMARD and with an IR to $\geq 1$ TNFi. Patients were randomized $2: 2: 1: 1$ to receive tofacitinib $5 \mathrm{mg}$ BID, tofacitinib $10 \mathrm{mg}$ BID, placebo $\rightarrow$ tofacitinib $5 \mathrm{mg}$ BID or placebo $\rightarrow$ tofacitinib $10 \mathrm{mg}$ BID. Patients on placebo switched to tofacitinib at month 3 [21].

Both studies included identical efficacy assessments at the same time points up to month 6.

OPAL Broaden and OPAL Beyond were conducted in accordance with the Good Clinical Practice Guidelines of the International Conference on Harmonisation, the Declaration of Helsinki, and the local country regulations. The study protocols were approved by the institutional review board or the independent ethics committee at each site.

\section{Efficacy Endpoints}

Endpoints included the proportions of patients achieving ACR20 response at month 3 (primary endpoint in the individual studies) and at time points other than month 3; proportions of patients achieving ACR50 and ACR70 responses at all time points; proportions of patients achieving the minimal clinically important difference for Health Assessment Questionnaire-Disability Index (HAQ-DI; range, 0-3; higher scores indicate greater disability) response (decrease from baseline of $\geq 0.35$ ) [23] in patients with baseline HAQ-DI $\geq 0.35$; proportions of patients achieving Psoriasis Area and Severity Index (PASI)75 $(\geq 75 \%$ improvement from baseline in PASI; range, 0.0-72.0; higher scores indicate more severe psoriasis) in patients with baseline body surface area (BSA; range, 0-100\%; higher scores indicate greater BSA affected by psoriasis) $\geq 3 \%$ and PASI $>0$; proportions of patients with resolution of enthesitis (Leeds Enthesitis Index [LEI] score of 0 ; range, $0-6$; higher scores indicate more affected sites) in patients with baseline LEI $>0$; proportions of patients with resolution of enthesitis (Spondyloarthritis Research Consortium of Canada [SPARCC] Enthesitis Index score of 0 ; range, $0-16$; higher scores indicate more affected sites) in patients with baseline SPARCC Enthesitis Index $>0$; proportions of patients with resolution of dactylitis (Dactylitis Severity Score [DSS] of 0; range, 0-60; higher scores indicate greater severity/more affected sites) in patients with baseline DSS $>0$ and proportions of patients with Bath Ankylosing Spondylitis Disease Activity Index (BASDAI) response (BASDAI $<4 \mathrm{~cm}$; range, $\quad 0-10 \mathrm{~cm}$; higher scores indicate more severe ankylosing spondylitis disease activity, including worse symptoms of back pain) in patients with presence of spondylitis as determined by the investigator at screening and with baseline BASDAI $\geq 4 \mathrm{~cm}$ (imaging was not required to confirm the presence of spondylitis). Other endpoints included the changes from baseline in the following manifestations of PsA: HAQ-DI at month 3 (primary endpoint in the individual studies) and at time points other than month 3 ; painful/tender joint count (JC; out of 68 joints; range, 0-68; higher score indicates a greater number of painful joints); swollen JC (out of 66 joints; range, 0-66; higher score indicates a greater number of inflamed joints); Dermatology Life Quality Index (DLQI; range, 0-30; higher scores indicate greater impairment); LEI in patients with baseline LEI $>0$; SPARCC Enthesitis Index in patients with baseline SPARCC Enthesitis Index $>0$; DSS in patients with baseline DSS $>0$; and BASDAI in patients with presence of spondylitis as determined by the investigator at screening and with baseline BASDAI $>0 \mathrm{~cm}$ and $\geq 4 \mathrm{~cm}$.

Most efficacy endpoints were assessed at week 2 and months 1, 2, 3, 4 and 6; psoriasis, enthesitis, dactylitis, and BASDAI were assessed at months 1, 3, and 6 .

All analyses were performed using the Full Analysis Set (FAS), which included all patients who were randomized and received $\geq 1$ dose of the study drug. For continuous endpoints for which change from baseline was assessed, a baseline value and $\geq 1$ post-baseline value were required for inclusion into the FAS for that 
endpoint. For endpoints such as psoriasis, enthesitis, dactylitis, or BASDAI, a subset of FAS was used, as there were no mandatory inclusion criteria relating to these endpoints for patients entering these studies, and therefore not all patients presented with the relevant affected domains.

Analyses at month 3 included patients randomized to the tofacitinib and placebo treatment groups only, with data from the two placebo sequences combined. Data for the adalimumab treatment group in OPAL Broaden have been reported previously [20] and are included in the supplementary material (Table S2) for comparison with the pooled analysis reported here; they were not included in the pooled analysis since there was no matching adalimumab group in OPAL Beyond. Analyses after month 3 included the tofacitinib groups only, since patients randomized to the placebo sequences were switched to tofacitinib after month 3; for this same reason, treatment comparisons between each tofacitinib dose and placebo were made at each visit to month 3 only.

For binary endpoints (ACR20/50/70 response rates, HAQ-DI response rate, PASI75 response rate, enthesitis resolution, dactylitis resolution, and BASDAI response rate), the difference in response proportions across studies was estimated using the Cochran-Mantel-Haenszel approach adjusting for study. Large sample approximation was used for statistical testing and for generating 95\% confidence intervals (CI). Non-response imputation (NRI) was applied, with missing response treated as nonresponse.

Changes from baseline in HAQ-DI, painful/tender JC, swollen JC, DLQI, LEI, SPARCC Enthesitis Index, DSS, and BASDAI were analyzed with a mixed model for repeated measures. The model included treatment, visit, treatment-by-visit interaction, geographic region, study and baseline value as fixed effects, and used a common unstructured variancecovariance matrix. Two separate analyses were performed; for analyses to month 3, placebo treatment sequences were combined into a single placebo group (results to month 3 are from this model), whereas for analyses to month 6 (including all post-baseline data to month 6; results after month 3 are from this model), only patients randomized to the tofacitinib groups are included. Missing values were not imputed.

Nominal $p$ values (or two-sided 95\% CI) were reported; as this is a post hoc analysis, there was no correction for multiplicity.

\section{RESULTS}

\section{Patient Disposition}

Of the 710 patients included in this analysis, 316 were from OPAL Broaden and 394 were from OPAL Beyond. Overall, 238, 236, and 236 patients received tofacitinib $5 \mathrm{mg}$ BID, tofacitinib $10 \mathrm{mg}$ BID, and placebo, respectively (Table 1). An additional 106 patients were randomized to adalimumab in OPAL Broaden, as previously reported [20]. By month 3, 4.6, 4.7, and $8.5 \%$ of patients receiving tofacitinib $5 \mathrm{mg}$ BID, tofacitinib $10 \mathrm{mg}$ BID, and placebo, respectively, discontinued from the studies. Discontinuations were primarily due to adverse events, insufficient clinical response, or patients no longer being willing to participate in the study.

\section{Patient Demographics and Baseline Characteristics}

The demographics and baseline characteristics of the pooled dataset from OPAL Broaden and OPAL Beyond were comparable between treatment groups (Table 1; Table S1 in the supplementary material). The majority of patients had polyarticular disease $(98.0 \%)$, psoriasis affecting $\geq 3 \%$ BSA (67.7\%), enthesitis (80.3\%), dactylitis $(52.5 \%)$, and high-sensitivity C-reactive protein levels above the upper limit of normal (> $2.87 \mathrm{mg} / \mathrm{l} ; 62.5 \%)$ at baseline. Of the TNFi-experienced patients, $18.0 \%$ and $13.2 \%$ had previously failed 2 and $\geq 3$ TNFi treatments, respectively. Methotrexate was the concomitant treatment for $78.7 \%$ of patients. Patients with an IR to TNFi had longer mean PsA durations vs. TNFi-naïve patients (mean 
Table 1 Demographics and baseline characteristics; pooled data from OPAL Broaden and OPAL Beyond

\begin{tabular}{|c|c|c|c|c|}
\hline & $\begin{array}{l}\text { Tofacitinib } 5 \text { mg BID } \\
(N=238)\end{array}$ & $\begin{array}{l}\text { Tofacitinib } 10 \mathrm{mg} \text { BID } \\
(N=236)\end{array}$ & $\begin{array}{l}\text { Placebo } \\
(N=236)\end{array}$ & $\begin{array}{l}\text { Total } \\
(N=710)\end{array}$ \\
\hline Age (years), mean (SD) & $49.5(12.4)$ & $49.4(11.7)$ & $48.4(12.5)$ & $49.1(12.2)$ \\
\hline Female, $n(\%)$ & $121(50.8)$ & $136(57.6)$ & $136(57.6)$ & $393(55.4)$ \\
\hline BMI $\left(\mathrm{kg} / \mathrm{m}^{2}\right)$, mean $(\mathrm{SD})$ & $29.8(6.3)$ & $30.2(6.3)$ & $29.2(5.6)$ & $29.7(6.1)$ \\
\hline Race, Caucasian ${ }^{\mathrm{a}}, n(\%)$ & $226(95.0)$ & $221(93.6)$ & $222(94.1)$ & $669(94.2)$ \\
\hline PsA duration (years), mean (SD) & $8.6(7.9)$ & $7.5(6.6)$ & $8.1(7.5)$ & $8.0(7.4)$ \\
\hline Tender $\mathrm{JC}^{\mathrm{b}}$, mean (SD) & $20.5(12.8)$ & $23.2(15.8)$ & $20.2(14.6)$ & $21.3(14.5)$ \\
\hline Swollen JC ${ }^{\mathrm{c}}$, mean $(\mathrm{SD})$ & $12.5(10.3)$ & $12.3(9.8)$ & $10.9(8.9)$ & $11.9(9.7)$ \\
\hline hsCRP $>2.87 \mathrm{mg} / \mathrm{l}, n(\%)$ & $153(64.3)$ & $148(62.7)$ & $143(60.6)$ & $444(62.5)$ \\
\hline Polyarticular disease ${ }^{\mathrm{d}}, n(\%)$ & $236(99.2)$ & $231(97.9)$ & $229(97.0)$ & $696(98.0)$ \\
\hline $\begin{array}{l}\text { Screening distal interphalangeal joints } \\
\text { involvement, } n(\%)\end{array}$ & $153(64.3)$ & $151(64.0)$ & $134(56.8)$ & $438(61.7)$ \\
\hline Arthritis mutilans, $n(\%)$ & $16(6.7)$ & $18(7.6)$ & $23(9.7)$ & $57(8.0)$ \\
\hline Spondylitis ${ }^{\mathrm{e}}, n(\%)$ & $50(21.0)$ & $47(19.9)$ & $44(18.6)$ & $141(19.9)$ \\
\hline Psoriatic BSA $\geq 3 \%, n(\%)$ & $162(68.1)$ & $151(64.0)$ & $168(71.2)$ & $481(67.7)$ \\
\hline \multirow[t]{2}{*}{ PASI $^{\mathrm{f}}$, mean $(\mathrm{SD})$} & $N 1=162$ & $N 1=151$ & $N 1=168$ & $N 1=481$ \\
\hline & $9.0(7.8)$ & $10.1(7.9)$ & $10.3(9.9)$ & $9.8(8.6)$ \\
\hline Enthesitis assessed by $\mathrm{LEI}^{\mathrm{g}}, n(\%)$ & $158(66.4)$ & $163(69.1)$ & $158(66.9)$ & $479(67.5)$ \\
\hline \multirow[t]{2}{*}{ LEI score (continuous) ${ }^{\mathrm{h}}$, mean $(\mathrm{SD})$} & $N 1=158$ & $N 1=163$ & $N 1=158$ & $N 1=479$ \\
\hline & $2.8(1.5)$ & $3.2(1.7)$ & $2.8(1.5)$ & $2.9(1.6)$ \\
\hline Enthesitis assessed by SPARCC ${ }^{\mathrm{i}}, n(\%)$ & $177(74.4)$ & $189(80.1)$ & $179(75.8)$ & $545(76.8)$ \\
\hline \multirow{2}{*}{ 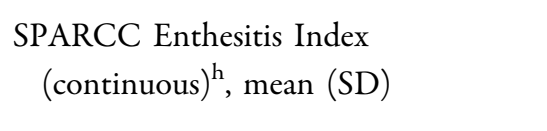 } & $N 1=177$ & $N 1=189$ & $N 1=179$ & $N 1=545$ \\
\hline & $5.4(3.7)$ & $6.1(4.2)$ & $5.3(3.6)$ & $5.6(3.9)$ \\
\hline Dactylitis ${ }^{\mathrm{j}}, n(\%)$ & $127(53.4)$ & $125(53.0)$ & $121(51.3)$ & $373(52.5)$ \\
\hline \multirow[t]{2}{*}{ DSS score (continuous) ${ }^{\mathrm{h}}$, mean $(\mathrm{SD})$} & $N 1=127$ & $N 1=125$ & $N 1=121$ & $N 1=373$ \\
\hline & $8.4(9.0)$ & $9.0(8.2)$ & $8.3(7.3)$ & $8.6(8.2)$ \\
\hline Baseline BASDAI $\geq 4 \mathrm{~cm}^{\mathrm{e}}, n(\%)$ & $43(18.1)$ & $41(17.4)$ & $38(16.1)$ & $122(17.2)$ \\
\hline \multirow[t]{2}{*}{ Baseline BASDAI ${ }^{\mathrm{e}}(\mathrm{cm})$, mean $(\mathrm{SD})$} & $N 1=50$ & $N 1=47$ & $N 1=44$ & $N 1=141$ \\
\hline & $6.0(2.0)$ & $6.3(2.1)$ & $6.5(2.0)$ & $6.3(2.0)$ \\
\hline Concomitant $\mathrm{MTX}^{\mathrm{k}}, n(\%)$ & $186(78.2)$ & $180(76.3)$ & $193(81.8)$ & $559(78.7)$ \\
\hline Corticosteroid use, $n(\%)$ & $67(28.2)$ & $37(15.7)$ & $49(20.8)$ & $153(21.5)$ \\
\hline Prior TNFi use, $n(\%)$ & $131(55.0)$ & $132(55.9)$ & $132(55.9)$ & $395(55.6)$ \\
\hline
\end{tabular}


Table 1 continued

\begin{tabular}{lllll}
\hline & $\begin{array}{l}\text { Tofacitinib } 5 \text { mg BID } \\
(N=238)\end{array}$ & $\begin{array}{l}\text { Tofacitinib } 10 \text { mg BID } \\
(N=236)\end{array}$ & $\begin{array}{l}\text { Placebo } \\
(\boldsymbol{N}=\mathbf{2 3 6})\end{array}$ & $\begin{array}{l}\text { Total } \\
(\boldsymbol{N}=710)\end{array}$ \\
\hline $\begin{array}{l}\text { Prior non-TNFi bDMARDs use } \\
n(\%)\end{array}$ & $14(5.9)$ & $18(7.6)$ & $14(5.9)$ & $46(6.5)$ \\
\hline
\end{tabular}

Additional characteristics are in Table $\mathrm{S} 1$ in the supplementary material

$B A S D A I$ Bath Ankylosing Spondylitis Disease Activity Index, $b D M A R D$ biologic disease-modifying antirheumatic drug, $B I D$ twice daily, $B M I$ body mass index, $B S A$ body surface area, csDMARD conventional synthetic disease-modifying antirheumatic drug, DSS Dactylitis Severity Score, FAS Full Analysis Set, $b s C R P$ high-sensitivity C-reactive protein, JC joint count, $L E I$ Leeds Enthesitis Index, $M T X$ methotrexate, $n$ number of patients meeting criterion, $N$ number of patients in FAS, $N 1$ number of evaluable patients, PASI Psoriasis Area and Severity Index, Ps $A$ psoriatic arthritis, $S D$ standard deviation, SPARCC Spondyloarthritis Research Consortium of Canada, TNFi tumor necrosis factor inhibitor

${ }^{a}$ Others were black, Asian, and other races

b Out of 68 joints

c Out of 66 joints

$\mathrm{d} \geq 5$ tender or swollen joints

e Patients with spondylitis (determined by the investigator site's qualified assessor) at screening

f Patients with baseline BSA $\geq 3 \%$ and baseline PASI $>0$

g Determined by baseline LEI $>0$

h Patients with baseline score $>0$

i Determined by baseline SPARCC Enthesitis Index $>0$

j Determined by baseline DSS $>0$

k MTX only; other patients received csDMARDs including sulfasalazine, leflunomide, and hydroxychloroquine

${ }^{1}$ Oral corticosteroid use at baseline

m Included patients who received any non-TNFi bDMARD or both TNFi and non-TNFi bDMARDs; non-TNFi bDMARDs included abatacept, anakinra, guselkumab, ixekizumab, rituximab, secukinumab, tocilizumab, and ustekinumab

[standard deviation]: 9.4 [7.5] vs. 6.1 [6.5] years). Across the treatment groups, $18.6-21.0 \%$ of patients had spondylitis symptoms at screening, and $2.5-2.9 \%$ and $16.1-18.1 \%$ of patients had baseline BASDAI $>0$ to $<4 \mathrm{~cm}$ and $\geq 4 \mathrm{~cm}$, respectively.

\section{Peripheral Arthritis}

ACR20 (a primary endpoint of each study), ACR50 and ACR70 response rates were higher with tofacitinib 5 and $10 \mathrm{mg}$ BID vs. placebo at month 3 ( $p \leq 0.05$; Fig. 1). ACR20, ACR50, and ACR70 response rates further improved or were maintained at month 6 (Fig. 2).

Improvement in physical function, assessed by change in HAQ-DI (primary endpoint of each study), was greater at month 3 with tofacitinib 5 and $10 \mathrm{mg}$ BID vs. placebo $(p<0.001$, Fig. 3a). A greater proportion of tofacitinib-treated patients achieved a clinically significant HAQ-DI response (decrease from baseline of $\geq 0.35$ ) at month 3 vs. placebo $(p<0.001$, Table 2$)$. Improvements in the number of painful/tender and swollen joints were also greater at month 3 with tofacitinib vs. placebo $(p<0.001$, Fig. $3 \mathrm{~b}$ and $3 \mathrm{c})$. Improvements were maintained to month 6 .

\section{Psoriasis}

A greater proportion of patients receiving tofacitinib 5 and $10 \mathrm{mg}$ BID achieved PASI75 vs. placebo at month 3 ( $p<0.001$; Fig. 3d); PASI75 response further improved at month 6 . PASI75 response was numerically greater in patients receiving tofacitinib 10 vs. $5 \mathrm{mg}$ BID. Patients receiving tofacitinib also achieved greater improvements in DLQI at month 3 vs. those receiving placebo $(p<0.001 ;$ Fig. $3 \mathrm{e})$, and greater improvements with tofacitinib 10 vs. 


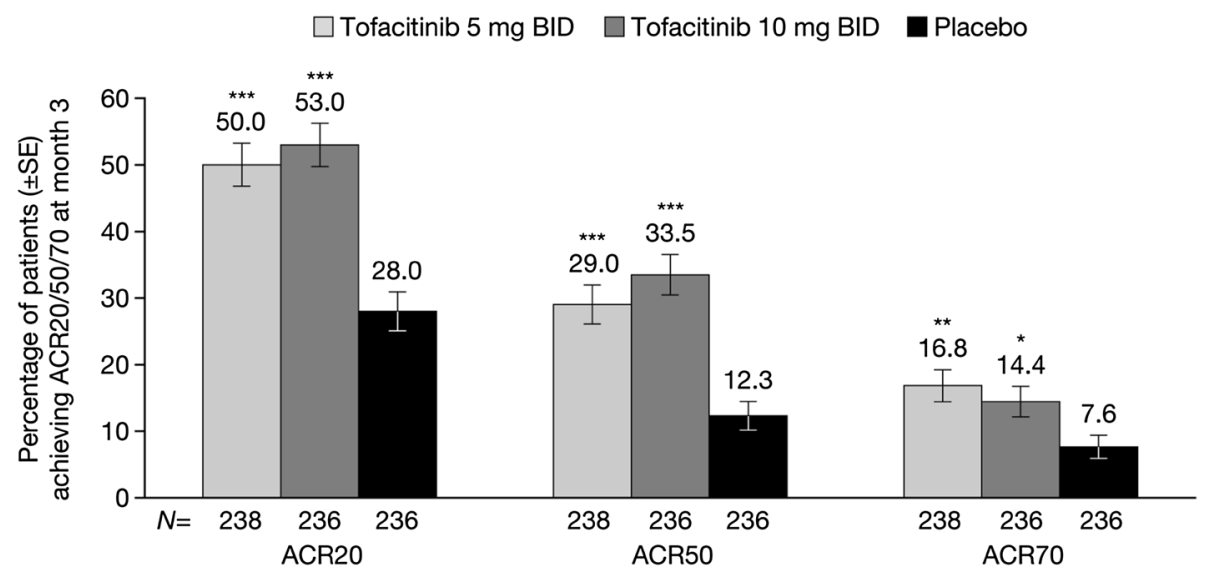

Fig. 1 ACR20, ACR50, and ACR70 response rates at month 3; pooled data from OPAL Broaden and OPAL Beyond (FAS, NRI). ACR American College of Rheumatology, BID twice daily, FAS Full Analysis Set, NRI nonresponse imputation, $S E$ standard error. ${ }^{*} p \leq 0.05$;

$5 \mathrm{mg}$ BID, with improvements increased or maintained at month 6 .

\section{Enthesitis and Dactylitis}

Changes in LEI and SPARCC Enthesitis Index were greater at month 3 for both tofacitinib doses vs. placebo ( $p<0.01$; Fig. $4 \mathrm{a}, \mathrm{b})$. A higher proportion of tofacitinib-treated patients achieved enthesitis resolution at month 3 , as measured by the LEI and SPARCC Enthesitis Index, vs. placebo $(p \leq 0.05$ except for tofacitinib $5 \mathrm{mg}$ BID for SPARCC Enthesitis Index; Table 2). Further improvements in all enthesitis endpoints were seen at month 6 .

The change in DSS, and the proportion of patients who achieved dactylitis resolution, were greater for tofacitinib vs. placebo at month 3 ( $p \leq 0.05$; Fig. $4 c$, Table 2$)$. Further improvements in both dactylitis endpoints were seen at month 6 .

\section{BASDAI}

In patients determined by the investigator as having spondylitis (although imaging was not mandated) at screening and baseline BASDAI $>0 \mathrm{~cm}$ or $\geq 4 \mathrm{~cm}$, changes in BASDAI at month 3 were greater vs. placebo with
${ }^{* *} p<0.01 ;{ }^{* * *} p<0.001$ vs. placebo; $p$ values are based on large sample approximation to difference in binomial proportions adjusting for study by CochranMantel-Haenszel approach; missing response was imputed as non-response

tofacitinib $10 \mathrm{mg}$ BID ( $p \leq 0.05$; Fig. $4 \mathrm{~d}$, e). BASDAI response rates were also greater vs. placebo with tofacitinib $5 \mathrm{mg}$ BID at month 3 $(p \leq 0.05$; Table 2$)$. Improvements were maintained at month 6 with both tofacitinib doses.

\section{Comparison with Findings from Primary Studies}

Endpoints for OPAL Broaden and OPAL Beyond are shown in detail in Table $\mathrm{S} 2$ in the supplementary material, reporting both nominal significance vs. placebo and significance vs. placebo under type 1 error control. ACR20, ACR50, and HAQ-DI response rates, and changes in HAQ-DI and swollen JC showed significant improvement $(p \leq 0.05)$ at month 3 with both doses of tofacitinib vs. placebo in the pooled dataset and in both the individual OPAL Broaden and OPAL Beyond studies (significance under type 1 error control for ACR20 and ACR50 response rates, and change in HAQ-DI in both individual studies). ACR70 response rates (significance under type 1 error control for both tofacitinib doses in OPAL Broaden), PASI75 response rates (significance under type 1 error control with both tofacitinib doses in OPAL Broaden and tofacitinib $10 \mathrm{mg}$ BID in OPAL Beyond), enthesitis (significance under type 1 error control for change in LEI with tofacitinib 


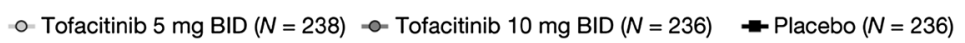

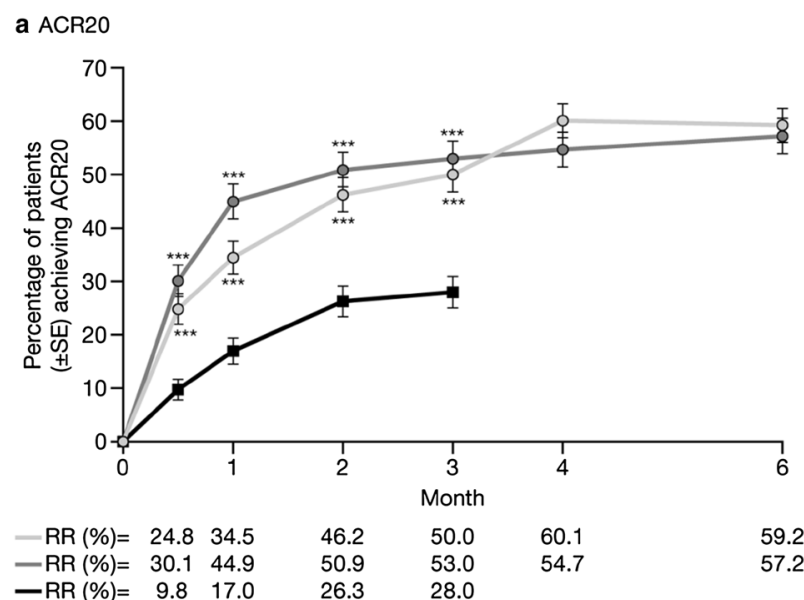

b ACR50
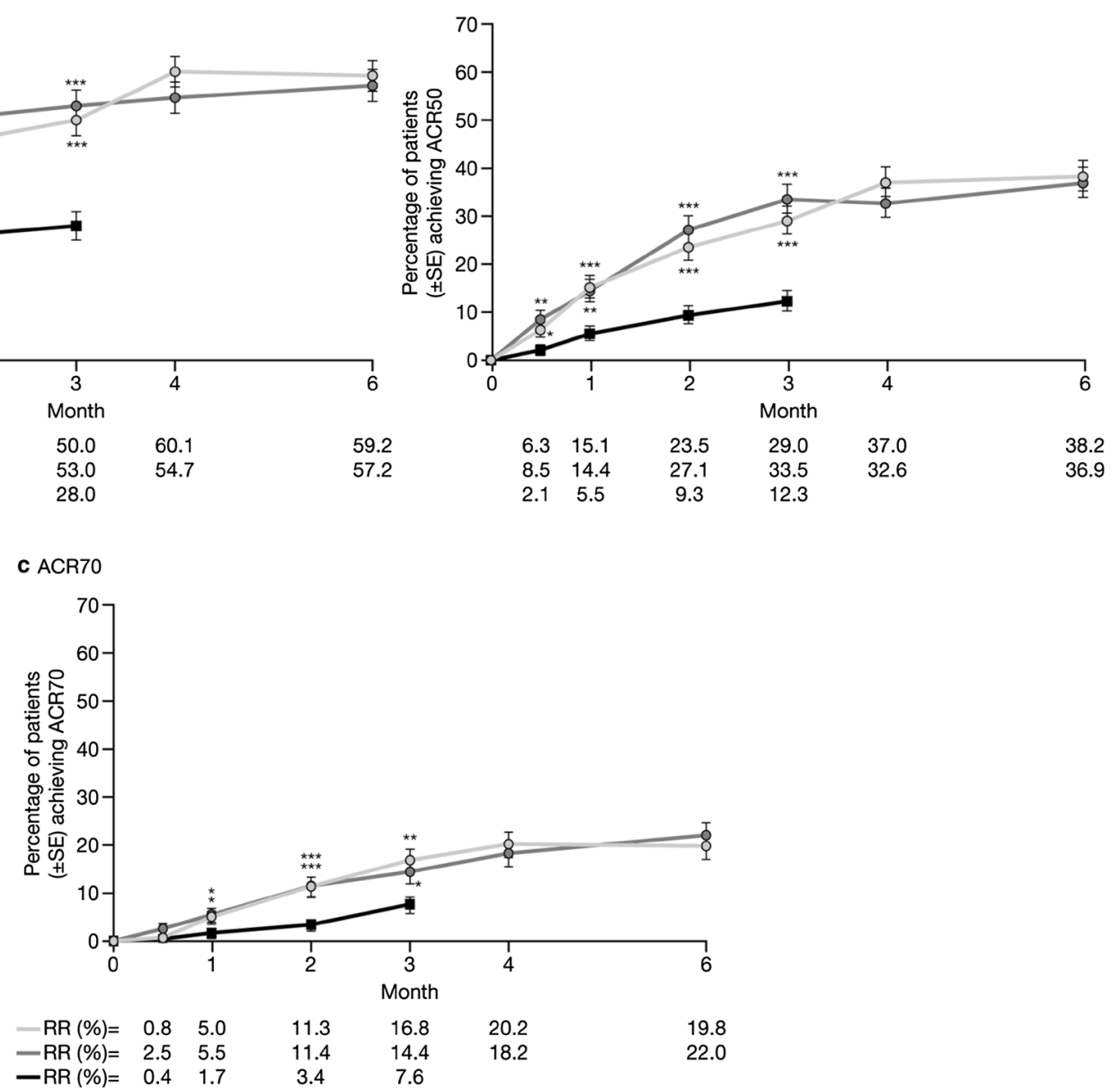

Fig. 2 ACR20, ACR50, and ACR70 response rates to month 6; pooled data from OPAL Broaden and OPAL Beyond (FAS, NRI). ACR American College of Rheumatology, BID twice daily, FAS Full Analysis Set, NRI nonresponse imputation, $R R$ response rate in terms of ACR response, $S E$ standard error. ${ }^{*} p \leq 0.05 ;{ }^{* *} p<0.01$;

$10 \mathrm{mg}$ BID in OPAL Broaden), dactylitis and change in painful/tender JC at month 3 reached statistical significance $(p \leq 0.05)$ with both doses vs. placebo in the pooled analysis, and with at least one dose in at least one of the individual studies.

\section{DISCUSSION}

This post hoc analysis of pooled data from two phase 3 studies of tofacitinib in patients with
${ }^{* * *} p<0.001$ vs. placebo; $p$ values are based on large sample approximation to difference in binomial proportions adjusting for study by Cochran-Mantel-Haenszel approach; $p$ values not calculated beyond month 3 as the placebo-controlled period ended at month 3; missing response was imputed as non-response

active PsA explored the efficacy of tofacitinib over 6 months of treatment. Patients included those who were naive to TNFi treatment (OPAL Broaden) and those with an IR to TNFi (OPAL Beyond), $13.2 \%$ of whom had received $\geq 3$ TNFi treatments; all were receiving one background csDMARD. Pooling has the benefit of increasing the sample size to obtain more precise estimates of the efficacy of a treatment, even in a population with mixed treatment history, especially for endpoints for disease manifestations that do not affect all patients. Furthermore, these 
results are particularly robust, as NRI was used in the analysis of binary endpoints; missing responses were imputed as non-responses, thus providing conservative estimates of response to treatment. The results of the pooled analyses are not intended to supersede any of the results of the pre-specified analyses in the individual studies; indeed, some efficacy outcomes, such as ACR response rates, tended to be lower for TNFiIR patients in OPAL Beyond compared with TNFi-naïve patients in OPAL Broaden [20, 21].

ACR20 responses with both tofacitinib doses at month 3 (50.0-53.0\%) and month 6 (57.2-59.2\%) were generally comparable with those reported for the adalimumab control in OPAL Broaden (51.9\% at month 3 and $64.2 \%$ at month 6) [20], as well as biologic DMARD and TNFi treatments for mixed populations of TNFinaïve and experienced patients in published studies (43.8-63.8\% across 3 and 6 months) $[8,9,11,12,24]$.

Physical function significantly improved $(p \leq 0.05)$ with both doses of tofacitinib vs. placebo at month 3 (change in HAQ-DI: -0.38 vs. -0.16$)$, as did the change in the number of painful/tender ( -9.6 to -10.4 vs. -5.8$)$ and swollen joints ( -7.2 vs. -3.7 ). At month 3 and month $6,44.7-56.1 \%$ of the tofacitinib-treated patients achieved clinically relevant improvements in HAQ-DI (decrease from baseline in HAQ-DI $\geq 0.35$ ). Additionally, a significantly greater proportion of tofacitinib-treated patients achieved PASI75 response at month 3 vs. placebo $(32.1-43.7 \%$ vs. $14.3 \% ; p \leq 0.05)$; this increased to month 6. Patients who received tofacitinib also experienced a significant $(p \leq 0.05)$ decrease from baseline in DLQI at month 3 vs. placebo, indicating improvements in quality of life (which was numerically greater with tofacitinib 10 vs. $5 \mathrm{mg}$ BID); this was maintained to month 6 . Improvements in enthesitis, dactylitis, and BASDAI scores with tofacitinib treatment vs. placebo were also observed at month 3 . The proportions of patients receiving tofacitinib 5 and $10 \mathrm{mg}$ BID at month 6 who achieved enthesitis (based on LEI and SPARCC) and dactylitis resolution were $39.0-47.5 \%$ and $55.9-60.8 \%$, respectively. The resolution of these clinical manifestations, which are associated with increased disease burden of PsA [25], is of particular importance as treatment options for these are limited $[26,27]$. Overall, a significant proportion of patients achieved ACR20, ACR50, ACR70, PASI75, and HAQ-DI responses, and improvements in enthesitis, dactylitis, and BASDAI scores were observed over time, indicating that tofacitinib treatment provides clinically meaningful improvements in the disease outcomes of PsA. There were no observed differences between the efficacy of tofacitinib 5 and $10 \mathrm{mg}$ BID in the peripheral arthritis, dactylitis, and enthesitis domains; however, rates of PASI75 and improvements in DLQI appeared to be greater with tofacitinib 10 vs. $5 \mathrm{mg}$ BID. While we report results for tofacitinib vs. placebo across multiple clinical outcomes, due to the heterogeneity of clinical manifestations of PsA, composite outcome measures assessing efficacy across the multiple domains of PsA in one single instrument may prove useful in future clinical trials [28].

Comparisons with the individual results from OPAL Broaden and OPAL Beyond showed generally consistent findings with this pooled analysis (Table $\mathrm{S} 2$ in the supplementary material), with ACR20, ACR50, and HAQ-DI response rates, changes in HAQ-DI and swollen JC significantly greater with tofacitinib vs. placebo in both individual studies and the pooled dataset. PASI75, enthesitis, dactylitis, and BASDAI endpoints represented PsA domains that were present at baseline for only a subgroup of the study populations and were therefore undersized for the statistical analysis of the individual studies; however, pooling the datasets increased the sample size to improve the precision of the effect estimates and revealed a difference between tofacitinib and placebo at month 3 . In the pooled analysis, significant differences were observed in ACR70 response rates, PASI75 response rates and changes in painful/tender JC, LEI and DSS $(p$ $\leq 0.05)$ vs. placebo at month 3 with both tofacitinib doses; these were significant in at least one of the individual phase 3 studies, but were not significant with both tofacitinib doses in both phase 3 studies. Changes in BASDAI in patients assessed as having spondylitis at screening (imaging was not required to confirm 
o- Tofacitinib $5 \mathrm{mg}$ BID -o- Tofacitinib $10 \mathrm{mg}$ BID $=$ - Placebo

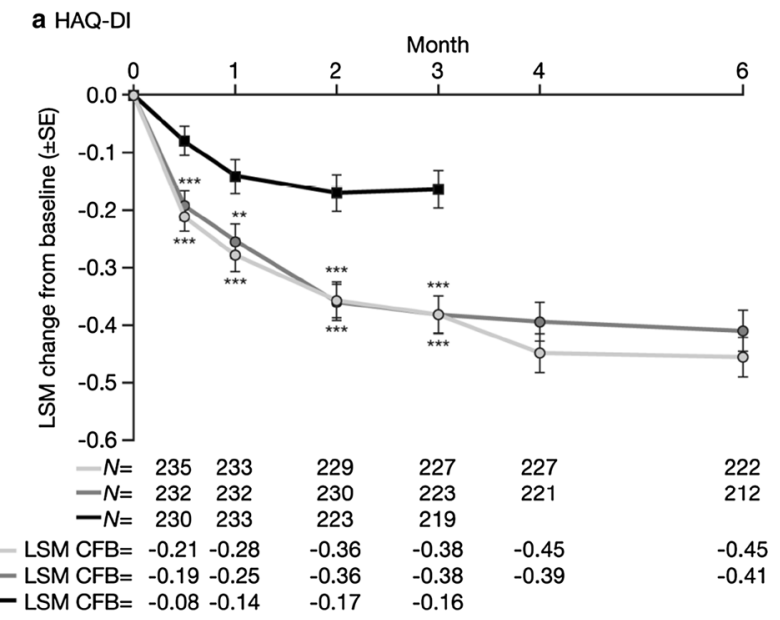

b Painful/tender $\mathrm{JC}^{\mathrm{a}}$

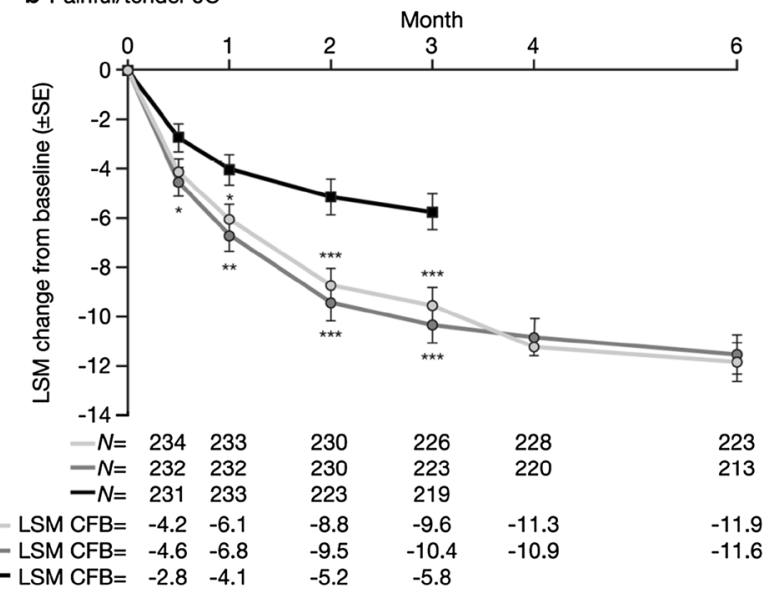

c Swollen $\mathrm{JC}^{\mathrm{b}}$

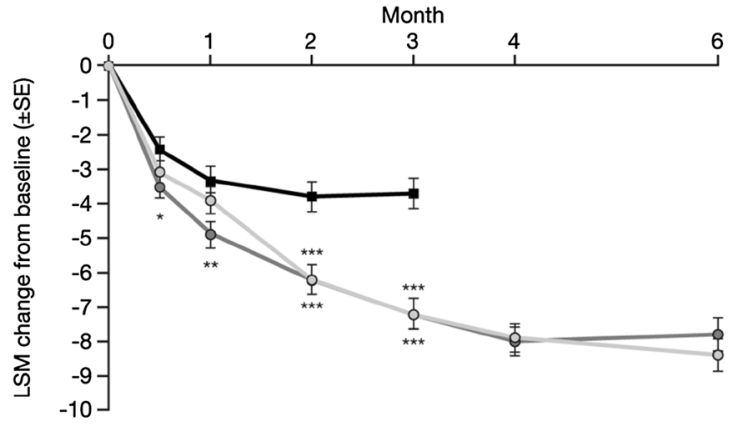

d PASI75

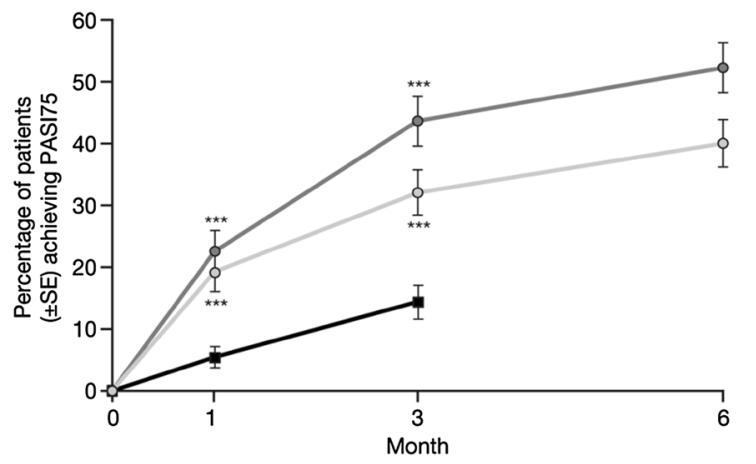

\begin{tabular}{|c|c|c|c|c|c|c|c|c|c|c|}
\hline$-N=$ & 234 & 233 & 230 & 226 & 228 & 223 & $-N=$ & 162 & 162 & 162 \\
\hline$-N=$ & 232 & 232 & 230 & 223 & 220 & 213 & $-N=$ & 151 & 151 & 151 \\
\hline$-N=$ & 231 & 233 & 223 & 219 & & & $-N=$ & 168 & 168 & \\
\hline- LSM CFB= & -3.1 & -3.9 & -6.2 & -7.2 & -7.9 & -8.4 & $-\mathrm{RR}(\%)=$ & 19.1 & 32.1 & 40.1 \\
\hline - LSM CFB= & -3.5 & -4.9 & -6.2 & -7.2 & -8.0 & -7.8 & $-\mathrm{RR}(\%)=$ & 22.5 & 43.7 & 52.3 \\
\hline - LSM CFB= & -2.4 & -3.3 & -3.8 & -3.7 & & & $-\mathrm{RR}(\%)=$ & 5.4 & 14.3 & \\
\hline
\end{tabular}

e DLQI

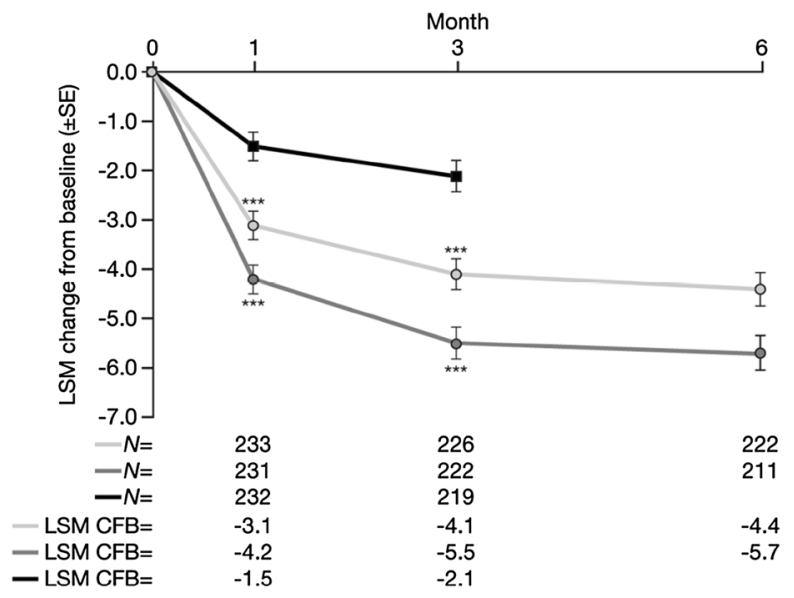


4 Fig. 3 LSM change from baseline in HAQ-DI, painful/tender JC and swollen JC, PASI75 response rate and LSM change from baseline in DLQI to month 6; pooled data from OPAL Broaden and OPAL Beyond. BID twice daily, BSA body surface area, $C F B$ change from baseline, $D L Q I$ Dermatology Life Quality Index, HAQ-DI Health Assessment Questionnaire-Disability Index, JC joint count, $L S M$ least squares mean, $P A S I$ Psoriasis Area and Severity Index, $R R$ response rate in terms of PASI75, SE standard error. ${ }^{*} p \leq 0.05 ;{ }^{* *} p<0.01 ;{ }^{* * *} p<0.001$ vs. placebo; $p$ values for HAQ-DI, painful/tender JC, swollen JC and DLQI are based on mixed model for repeated measures without imputation for missing values; $p$ values for PASI75 are based on large sample approximation to difference in binomial proportions adjusting for study by Cochran-MantelHaenszel approach; missing response was imputed as nonresponse; $p$ values not calculated beyond month 3 as the placebo-controlled period ended at month 3 . ${ }^{\text {a }}$ Out of 68 joints; ${ }^{\mathrm{b}}$ Out of 66 joints. $\mathrm{N}$ for HAQ-DI, painful/tender JC, swollen JC and DLQI is the number of patients evaluable at each visit; $\mathrm{N}$ for PASI75 is the number of patients with baseline $\mathrm{BSA} \geq 3 \%$ and PASI $>0$ the presence of spondylitis) and baseline BASDAI $>0 \mathrm{~cm}$ and $\geq 4 \mathrm{~cm}$ were only significant at month 3 with tofacitinib $10 \mathrm{mg}$ BID vs. placebo in the pooled analysis and OPAL Broaden (baseline BASDAI $>0 \mathrm{~cm}$ only; $p \leq 0.05$ ).

This analysis has a number of limitations. Although most of the pooled analyses were prespecified prior to unblinding of data, this was considered to be a post hoc analysis; OPAL Broaden and OPAL Beyond were designed to include study populations with distinctly different treatment histories, and therefore comparisons between the pooled analysis and the individual studies must be made with caution. Furthermore, comparisons with placebo were limited to the 3-month placebo-controlled portion of the phase 3 studies, and there was no stratification for background use of methotrexate [29]. Axial symptoms were assessed using BASDAI, but axial involvement was not an

Table 2 Physical function, enthesitis, dactylitis, and BASDAI endpoints at month 3 and month 6; pooled data from OPAL Broaden and OPAL Beyond (NRI)

\begin{tabular}{|c|c|c|c|c|c|}
\hline & \multicolumn{3}{|l|}{ Month 3} & \multicolumn{2}{|l|}{ Month 6} \\
\hline & $\begin{array}{l}\text { Tofacitinib } \\
5 \mathrm{mg} \text { BID }\end{array}$ & $\begin{array}{l}\text { Tofacitinib } \\
10 \mathrm{mg} \text { BID }\end{array}$ & Placebo & $\begin{array}{l}\text { Tofacitinib } \\
5 \mathrm{mg} \text { BID }\end{array}$ & $\begin{array}{l}\text { Tofacitinib } \\
10 \mathrm{mg} \text { BID }\end{array}$ \\
\hline $\begin{array}{l}\text { HAQ-DI response rate }{ }^{\mathrm{a}} \text {, } \\
\quad n / N(\%)\end{array}$ & $109 / 212^{* * *}(51.4)$ & $101 / 215^{* * *}(47.0)$ & $61 / 210(29.1)$ & $119 / 212(56.1)$ & $96 / 215(44.7)$ \\
\hline $\begin{array}{l}\text { Enthesitis resolution rate } \\
\quad(\text { LEI })^{\mathrm{b}}, n / N(\%)\end{array}$ & $58 / 158^{* *}(36.7)$ & $58 / 163^{* *}(35.6)$ & $34 / 158(21.5)$ & $75 / 158(47.5)$ & $71 / 163(43.6)$ \\
\hline $\begin{array}{l}\text { Enthesitis resolution rate } \\
\text { (SPARCC Enthesitis } \\
\text { Index) })^{\mathrm{b}}, n / N(\%)\end{array}$ & $52 / 177(29.4)$ & $66 / 189^{*}(34.9)$ & $42 / 179(23.5)$ & $69 / 177(39.0)$ & $76 / 189(40.2)$ \\
\hline $\begin{array}{l}\text { Dactylitis resolution rate } \\
\quad(\mathrm{DSS})^{\mathrm{b}}, n / N(\%)\end{array}$ & $55 / 127^{*}(43.3)$ & $69 / 125^{* * *}(55.2)$ & $37 / 121(30.6)$ & $71 / 127(55.9)$ & $76 / 125(60.8)$ \\
\hline $\begin{array}{l}\text { BASDAI response rate }{ }^{\mathrm{c}} \text {, } \\
\quad n / N(\%)\end{array}$ & $16 / 43^{*}(37.2)$ & $12 / 41(29.3)$ & $6 / 38(15.8)$ & $16 / 43(37.2)$ & $10 / 41(24.4)$ \\
\hline
\end{tabular}

BASDAI Bath Ankylosing Spondylitis Disease Activity Index, BID twice daily, DSS Dactylitis Severity Score, HAQ-DI Health Assessment Questionnaire-Disability Index, $L E I$ Leeds Enthesitis Index, $n$ number of patients with response, $N$ number of patients in the Full Analysis Set meeting baseline endpoint-specific criteria, NRI non-response imputation, SPARCC Spondyloarthritis Research Consortium of Canada

${ }^{*} p \leq 0.05 ;{ }^{* *} p<0.01 ;{ }^{* * *} p<0.001$ vs. placebo at month $3 ; p$ values are based on large sample approximation to difference in binomial proportions adjusting for study by Cochran-Mantel-Haenszel approach; $p$ values not calculated at month 6 as the placebo-controlled period ended at month 3; missing response was imputed as non-response

${ }^{a}$ Decrease from baseline of $\geq 0.35$ in HAQ-DI among patients with baseline HAQ-DI $\geq 0.35$

${ }^{\mathrm{b}}$ Indicated by post-baseline score $=0$ in patients with baseline score $>0$

${ }^{c}$ BASDAI $<4 \mathrm{~cm}$ among patients with spondylitis at screening and baseline BASDAI $\geq 4 \mathrm{~cm}$ 
○- Tofacitinib $5 \mathrm{mg}$ BID $\quad$ - Tofacitinib $10 \mathrm{mg}$ BID $\quad$-Placebo


d BASDAI
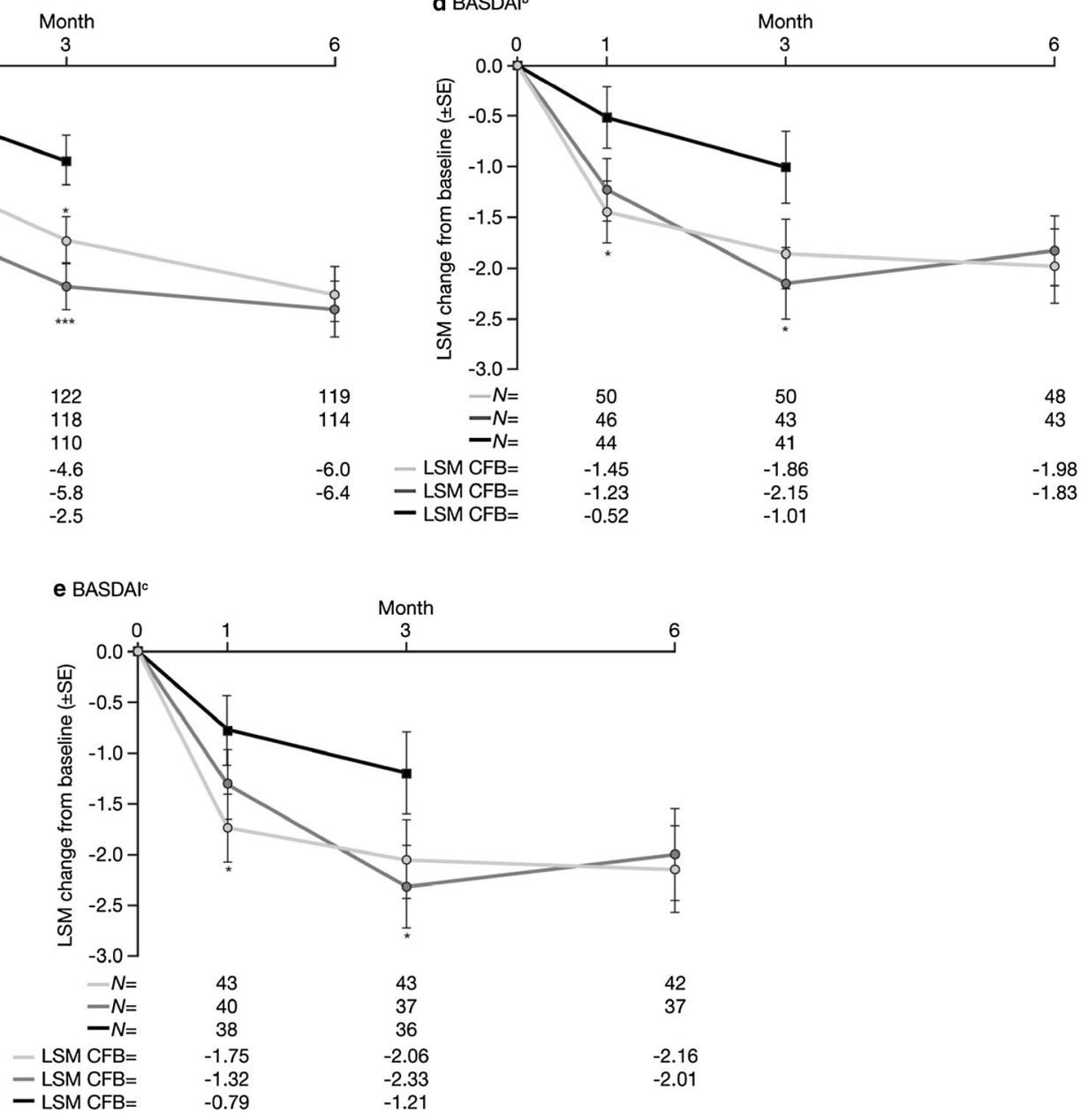
४Fig. 4 LSM change from baseline in LEI, SPARCC Enthesitis Index, DSS and BASDAI to month 6; pooled data from OPAL Broaden and OPAL Beyond. BASDAI Bath Ankylosing Spondylitis Disease Activity Index, BID twice daily, $C F B$ change from baseline, DSS Dactylitis Severity Score, LEI Leeds Enthesitis Index, LSM least squares mean, $N$ number of patients evaluable at each visit, $S E$ standard error, SPARCC Spondyloarthritis Research Consortium of Canada. $\quad{ }^{*} p \leq 0.05 ; \quad{ }^{* *} p<0.01$; ${ }^{* * *} p<0.001$ vs. placebo; $p$ values are based on mixed model for repeated measures without imputation for missing values; $p$ values not calculated beyond month 3 as the placebo-controlled period ended at month $3 .{ }^{a}$ Patients with baseline score $>0$; ${ }^{\mathrm{b}}$ Patients with spondylitis (as determined by the investigator site's qualified assessor) at screening and baseline BASDAI $>0 \mathrm{~cm}$; ${ }^{c}$ Patients with spondylitis (as determined by the investigator site's qualified assessor) at screening and baseline $\mathrm{BASDAI} \geq 4 \mathrm{~cm}$

inclusion criterion for the studies nor were rigorous diagnostic criteria defined in the protocols (imaging was not performed); a limited number of patients with axial symptoms were identified by qualified assessors at investigational sites. Additionally, with respect to the statistical analyses, $p$ values were generated with no correction for multiplicity. Finally, this analysis focuses on the efficacy of tofacitinib in treating PsA; safety data are reported in the primary manuscripts $[20,21]$ as well as in a pooled safety analysis [30]. No new safety risks were identified in an interim analysis of data from patients with active PsA receiving tofacitinib for up to 36 months in the ongoing LTE study, OPAL Balance (NCT01976364; datacut: November 2017; database not locked; data may change) [31].

\section{CONCLUSIONS}

In conclusion, in a pooled analysis of two PsA phase 3 trials of TNFi-naïve patients with an IR to csDMARDs and patients with an IR to TNFi, tofacitinib 5 and $10 \mathrm{mg}$ BID showed greater improvements vs. placebo at month 3 across four PsA disease domains: peripheral arthritis (including physical function), psoriasis, enthesitis and dactylitis, with efficacy maintained or improved at month 6 .

\section{ACKNOWLEDGEMENTS}

The authors thank the patients who participated in the OPAL Broaden and OPAL Beyond clinical studies.

Funding. This study, editorial support, and article processing charges were funded by Pfizer Inc. All authors had full access to all of the data in this study and take complete responsibility for the integrity of the data and accuracy of the data analysis.

Medical Writing Assistance. Medical writing support under the guidance of the authors was provided by Carole Evans, $\mathrm{PhD}$, on behalf of CMC Connect, a division of Complete Medical Communications Ltd, Macclesfield, UK, and Christina Viegelmann, PhD, at CMC Connect, a division of Complete Medical Communications Ltd, Glasgow, UK, and was funded by Pfizer Inc, New York, NY, USA in accordance with Good Publication Practice (GPP3) guidelines (Ann Intern Med 2015;163:461-464).

Authorship. All named authors meet the International Committee of Medical Journal Editors (ICMJE) criteria for authorship of this manuscript, take responsibility for the integrity of the work as a whole, and have given final approval to the version to be published.

Authorship Contributions. Peter Nash, Roy Fleischmann and Kim A. Papp were involved in the acquisition of the data. Cunshan Wang, Sujatha Menon, and William C. Ports were involved in the conception and design of the study. Cunshan Wang and Joseph Wu performed and are responsible for the statistical analyses. All authors were involved in data analysis, data interpretation, drafting the manuscript, and revising it critically, and have read and approved the final manuscript.

Prior presentation. These data have previously been presented in part at the European League Against Rheumatism (EULAR) 2017 congress (Ann Rheum Dis 2017;76(S2):951-952) and the American College of Rheumatology 
Annual Scientific Meeting 2017 (Arthritis Rheumatol 2017;69(S10):837-838).

Disclosures. Peter Nash has received grant/research support and honoraria for lectures and advice from AbbVie, Amgen, Bristol-Myers Squibb, Celgene, Eli Lilly, Janssen, Merck, Novartis, Pfizer Inc, Roche, Sanofi-Aventis and UCB. Laura C. Coates is a member of the speakers' bureau for Pfizer Inc. Roy Fleischmann has received grant/research support from Abbott, Amgen, Boehringer Ingelheim, Bristol-Myers Squibb, Celgene, Eli Lilly, Genentech, Janssen, Novartis, Pfizer Inc, Regeneron, Roche, Sanofi-Aventis and UCB, and is a consultant for Abbott, Amgen, Bristol-Myers Squibb, Eli Lilly, GSK, Janssen, Novartis, Pfizer Inc, Sanofi-Aventis and UCB. Kim A. Papp has received grant/research support from Abbott, Amgen, Anacor, Astellas, Celgene, Celtic, Dow Pharma, Eli Lilly, Galderma, Janssen, Janssen Biotech (Centocor), Merck, Novartis and Pfizer Inc, is a consultant for $3 \mathrm{M}$, Abbott, Akros, Alza, Amgen, Astellas, Baxter, Boehringer Ingelheim, Celgene, Cipher, Eli Lilly, Forward Pharma, Funxional Therapeutics, Galderma, Genentech, Isotechnika, Janssen, Janssen Biotech (Centocor), J\&J, Kirin, Kyowa, Meiji Seika Pharma, Merck, Mitsubishi Pharma, Mylan, Novartis, Pfizer Inc, Regeneron, Sanofi-Aventis, Serono, Stiefel, Takeda and UCB, and is a member of the speakers' bureau for $3 \mathrm{M}$, Abbott, Amgen, Astellas, Boehringer Ingelheim, Celgene, Eli Lilly, Galderma, Janssen, Merck, Novartis and Pfizer Inc. Juan Jesus Gomez-Reino has received grant/research support from AbbVie, Novartis, Pfizer Inc, Roche and UCB, is a consultant for Pfizer Inc, and is a member of the speakers' bureau for AbbVie, Bristol-Myers Squibb, Janssen, MSD, Pfizer Inc, Roche and UCB. Keith S. Kanik is a shareholder and employee of Pfizer Inc. Cunshan Wang is a shareholder and employee of Pfizer Inc. Joseph $\mathrm{Wu}$ is a shareholder and employee of Pfizer Inc. Sujatha Menon is a shareholder and employee of Pfizer Inc. Thijs Hendrikx was a shareholder and employee of Pfizer Inc at the time of this analysis, and is currently employed at Galapagos, Leiden, The Netherlands. William C. Ports is a shareholder and employee of Pfizer Inc.

Compliance with Ethics Guidelines. OPAL Broaden and OPAL Beyond were conducted in accordance with the Good Clinical Practice Guidelines of the International Conference on Harmonisation, the Declaration of Helsinki, and the local country regulations. The study protocols were approved by the institutional review board or the independent ethics committee at each site.

Data Availability. Upon request, and subject to certain criteria, conditions and exceptions (see https://www.pfizer.com/science/ clinical-trials/trial-data-and-results for more information), Pfizer will provide access to individual de-identified participant data from Pfizersponsored global interventional clinical studies conducted for medicines, vaccines, and medical devices (1) for indications that have been approved in the US and/or EU, or (2) in programmes that have been terminated (i.e., development for all indications has been discontinued). Pfizer will also consider requests for the protocol, data dictionary and statistical analysis plan. Data may be requested from Pfizer trials 24 months after study completion. The deidentified participant data will be made available to researchers whose proposals meet the research criteria and other conditions, and for which an exception does not apply, via a secure portal. To gain access, data requestors must enter into a data access agreement with Pfizer.

Open Access. This article is distributed under the terms of the Creative Commons Attribution-NonCommercial 4.0 International License (http://creativecommons.org/licenses/ by-nc/4.0/), which permits any noncommercial use, distribution, and reproduction in any medium, provided you give appropriate credit to the original author(s) and the source, provide a link to the Creative Commons license, and indicate if changes were made.

\section{REFERENCES}

1. Coates LC, Kavanaugh A, Mease PJ, et al. Group for Research and Assessment of Psoriasis and Psoriatic Arthritis 2015 treatment recommendations for psoriatic arthritis. Arthritis Rheumatol. 2016;68: 1060-71. 
2. Prey S, Paul C, Bronsard V, et al. Assessment of risk of psoriatic arthritis in patients with plaque psoriasis: a systematic review of the literature. J Eur Acad Dermatol Venereol. 2010;24(Suppl 2):31-5.

3. Mease PJ, Gladman DD, Papp KA, et al. Prevalence of rheumatologist-diagnosed psoriatic arthritis in patients with psoriasis in European/North American dermatology clinics. J Am Acad Dermatol. 2013;69:729-35.

4. López Estebaránz JL, Zarco-Montejo P, Samaniego ML, García-Calvo C. Prevalence and clinical features of psoriatic arthritis in psoriasis patients in Spain. Limitations of PASE as a screening tool. Eur J Dermatol. 2015;25:57-63.

5. Overman CL, Kool MB, da Silva JA, Geenen R. The prevalence of severe fatigue in rheumatic diseases: an international study. Clin Rheumatol. 2016;35:409-15.

6. Schwartzman S, Li Y, Zhou H, Palmer JB. Economic impact of biologic utilization patterns in patients with psoriatic arthritis. Clin Rheumatol. 2017;36:1579-88.

7. Kavanaugh A, Helliwell P, Ritchlin CT. Psoriatic arthritis and burden of disease: patient perspectives from the population-based Multinational Assessment of Psoriasis and Psoriatic Arthritis (MAPP) survey. Rheumatol Ther. 2016;3:91-102.

8. Kavanaugh A, Krueger GG, Beutler A, et al. Infliximab maintains a high degree of clinical response in patients with active psoriatic arthritis through 1 year of treatment: results from the IMPACT 2 trial. Ann Rheum Dis. 2007;66:498-505.

9. Mease PJ, Fleischmann R, Deodhar AA, et al. Effect of certolizumab pegol on signs and symptoms in patients with psoriatic arthritis: 24-week results of a phase 3 double-blind randomised placebo-controlled study (RAPID-PsA). Ann Rheum Dis. 2014;73:48-55.

10. Mease PJ, Gottlieb AB, van der Heijde $D$, et al. Efficacy and safety of abatacept, a T-cell modulator, in a randomised, double-blind, placebo-controlled, phase III study in psoriatic arthritis. Ann Rheum Dis. 2017;76:1550-8.

11. Ritchlin C, Rahman P, Kavanaugh A, et al. Efficacy and safety of the anti-IL-12/23 p40 monoclonal antibody, ustekinumab, in patients with active psoriatic arthritis despite conventional non-biological and biological anti-tumour necrosis factor therapy: 6-month and 1-year results of the phase 3, multicentre, double-blind, placebo-controlled, randomised PSUMMIT 2 trial. Ann Rheum Dis. 2014;73:990-9.
12. Mease PJ, McInnes IB, Kirkham B, et al. Secukinumab inhibition of interleukin-17A in patients with psoriatic arthritis. $\mathrm{N}$ Engl J Med. 2015;373:1329-39.

13. Kavanaugh A, Mease PJ, Gomez-Reino JJ, et al. Treatment of psoriatic arthritis in a phase 3 randomised, placebo-controlled trial with apremilast, an oral phosphodiesterase 4 inhibitor. Ann Rheum Dis. 2014;73:1020-6.

14. Mease PJ, Fleischmann RM, Wollenhaupt J, et al. Effect of certolizumab pegol over 48 weeks on signs and symptoms in patients with psoriatic arthritis with and without prior tumor necrosis factor inhibitor exposure. Arthritis Rheum. 2013;65(Supp 10): S132-3.

15. Coates LC, Helliwell PS. Validation of minimal disease activity criteria for psoriatic arthritis using interventional trial data. Arthritis Care Res (Hoboken). 2010;62:965-9.

16. Kavanaugh A, McInnes IB, Mease PJ, et al. Impact of persistent minimal disease activity on long-term outcomes in psoriatic arthritis: results from 5 years of the long-term extension of a randomized, placebo-controlled, study. Arthritis Rheum. 2013; 65(Supp 10):S147-8.

17. Mease PJ, Heckaman M, Kary S, Kupper H. Application and modifications of minimal disease activity measures for patients with psoriatic arthritis treated with adalimumab: subanalyses of ADEPT. J Rheumatol. 2013;40:647-52.

18. Bonafede M, Fox KM, Watson C, Princic N, Gandra SR. Treatment patterns in the first year after initiating tumor necrosis factor blockers in real-world settings. Adv Ther. 2012;29:664-74.

19. Costa L, Del Puente A, Peluso R, et al. Small molecule therapy for managing moderate to severe psoriatic arthritis. Expert Opin Pharmacother. 2017;18:1557-67.

20. Mease P, Hall S, Fitzgerald $\mathrm{O}$, et al. Tofacitinib or adalimumab versus placebo for psoriatic arthritis. N Engl J Med. 2017;377:1537-50.

21. Gladman D, Rigby W, Azevedo VF, et al. Tofacitinib for psoriatic arthritis in patients with an inadequate response to TNF inhibitors. $\mathrm{N}$ Engl $\mathrm{J}$ Med. 2017;377:1525-36.

22. Wang TS, Tsai TF. Tofacitinib in psoriatic arthritis. Immunotherapy. 2017;9:1153-63.

23. Mease PJ, Woolley JM, Bitman B, Wang BC, Globe DR, Singh A. Minimally important difference of Health Assessment Questionnaire in psoriatic arthritis: relating thresholds of improvement in 
functional ability to patient-rated importance and satisfaction. J Rheumatol. 2011;38:2461-5.

24. Mease PJ, Kivitz AJ, Burch FX, et al. Etanercept treatment of psoriatic arthritis: safety, efficacy, and effect on disease progression. Arthritis Rheum. 2004;50:2264-72.

25. Mease PJ, Karki C, Palmer JB, et al. Clinical characteristics, disease activity, and patient-reported outcomes in psoriatic arthritis patients with dactylitis or enthesitis: results from the Corrona Psoriatic Arthritis/Spondyloarthritis Registry. Arthritis Care Res (Hoboken). 2017;69:1692-9.

26. Rose S, Toloza S, Bautista-Molano W, Helliwell PS. Comprehensive treatment of dactylitis in psoriatic arthritis. J Rheumatol. 2014;41:2295-300.

27. Orbai AM, Weitz J, Siegel EL, et al. Systematic review of treatment effectiveness and outcome measures for enthesitis in psoriatic arthritis. J Rheumatol. 2014;41:2290-4.
28. Helliwell PS, FitzGerald O, Fransen J. Composite disease activity and responder indices for psoriatic arthritis: a report from the GRAPPA 2013 meeting on development of cutoffs for both disease activity states and response. J Rheumatol. 2014;41:1212-7.

29. Kivitz AJ, Fitzgerald O, Nash P, et al. Efficacy of tofacitinib by background methotrexate dose in patients with psoriatic arthritis: a post hoc analysis of pooled data from 2 phase 3 trials. Arthritis Rhematol. 2017;69(S10):830-1.

30. Burmester G, Fitzgerald O, Winthrop K, et al. Integrated safety summary of tofacitinib in psoriatic arthritis clinical studies. Ann Rheum Dis. 2017;76(S2):938.

31. Nash P, Coates LC, Kivitz AJ, et al. Safety and efficacy of tofacitinib, an oral Janus kinase inhibitor, up to 36 months in patients with active psoriatic arthritis: data from the third interim analysis of OPAL Balance, an open-label long-term extension study. Ann Rheum Dis. 2018;77:A1010. 